


\title{
BEIHEFTE
}

ZUR

ZEITSCHRIFT

FUR

\section{ROMANISCHE PHILOLOGIE}

\section{BEGRUNDET VON Prof. Dr. GUSTAV GROBER †}

\author{
FORTGEFUHRT UND HERAUSGEGEBEN
}

VON

DR. WALTHER v. WARTBURG

PROFESSOR AN DER UNIVERSITAT BASEL

\section{HEFT}

\section{IRENE BEHRENS}

DIE LEHRE VON DER EINTEILUNG DER DICHTKUNST VORNEHMLICH VOM 16. BIS 19. JAHRHUNDERT STUDIEN ZUR GESCHICHTE DER POETISCHEN GATTUNGEN

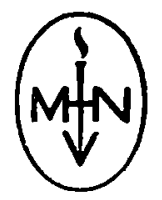

MAX NIEMEYER VERLAG / HALLE/SAALE 1940 


\section{DIE LEHRE VON DER EINTEILUNG DER DICHTKUNST}

VORNEHMLICH VOM 16. BIS 19. JAHRHUNDERT STUDIEN ZUR GESCHICHTE DER POETISCHEN GATTUNGEN

VON

IRENE BEHRENS

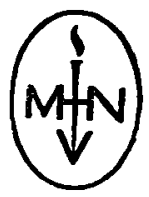

MAX NIEMEYER VERLAG / HALLE/SAALE 1940 
Alle Rechte,

auch das der Obersetzung in fremde Sprachen, vorbehalten Copyright by Max Niemejer Verlag, Halle (Saale), 1940

Printed in Germany

D 5

Buchdradkerei Richard Mayr, Würzburg 
Herrn Professor Dr. E. R. Curtius, meinem verehrten Lehrer,

danke ich für die Stellung des Themas und die Förderung dieses Buches.

Irene Behrens. 
